Case Report

\title{
Octreotide Use in a Patient with MEN-1 Syndrome and Multifocal Pancreatic Neuroendocrine Tumors: A Case Report and Review of the Literature
}

\author{
Matthew H. Kemm, Cory D. Manly, Thanh D. Hoang $(\mathbb{D}$, \\ Vinh Q. Mai, and Mohamed K. M. Shakir \\ Division of Endocrinology, Department of Medicine, Walter Reed National Military Medical Center, Bethesda, USA \\ Correspondence should be addressed to Thanh D. Hoang; tdhdthanh@gmail.com
}

Received 17 December 2018; Accepted 15 April 2019; Published 30 April 2019

Academic Editor: Yoshifumi Nakayama

Copyright (C) 2019 Matthew H. Kemm et al. This is an open access article distributed under the Creative Commons Attribution License, which permits unrestricted use, distribution, and reproduction in any medium, provided the original work is properly cited.

\begin{abstract}
We report a patient with multiple endocrine neoplasia type 1 with pancreatic polypeptide (PP) secreting subcentimeter pancreatic neuroendocrine tumors (pNETs) treated with octreotide and review the current literature that pertains to the management of these patients. Clinical data, laboratory results, and imaging were reviewed. A literature search was performed in PUBMED using combinations of the terms "multiple endocrine neoplasia type 1," "somatostatin," octreotide," "pancreatic polypeptide," and "pancreatic tumor." Relevant references were selected and reviewed. A 43-year-old male with a history of MEN1 and multiple subcentimeter neuroendocrine tumors with elevation of PP was treated with octreotide therapy leading to a reduction and normalization of PP levels. The patient tolerated octreotide therapy but self-discontinued octreotide after 24 months with a rise in PP levels off therapy. Tumors remained stable in size through 40 months of imaging follow-up. In patients with MEN1 and subcentimeter pNETs, octreotide therapy is well tolerated and can lead to a significant drop in PP levels with no change in lesion size. There is insufficient data to suggest long-term benefit with octreotide therapy but it may be considered versus standard conservative management.
\end{abstract}

\section{Introduction}

Multiple endocrine neoplasia type 1 (MEN1) is a rare, autosomal dominant syndrome causing a predisposition to tumors of the parathyroid glands, anterior pituitary, and enteropancreatic endocrine cells with an estimated prevalence of 2 to 20 per 100,000 individuals [1]. The malignant potential of pancreatic neuroendocrine tumors (pNETs) is now the primary life-threatening manifestation of MEN1 [2]. Functioning enteropancreatic endocrine tumors such as gastrinomas, insulinomas, or the rarely seen VIPomas or glucagonomas may be readily diagnosed based on clinical symptomatology alone [1]. However, nonfunctional tumors may constitute the bulk of these enteropancreatic tumors with studies suggesting a prevalence of $30-80 \%$ and with current screening protocols being likely discovered very early in the disease process $[3,4]$. These asymptomatic, enteropancreatic tumors may be malignant and capable of causing liver metastases with evidence to suggest a worsened prognosis compared to functional tumors.

Current recommendations for screening for enteropancreatic neuroendocrine tumors include both biochemical evaluation and radiological screening [1]. Cost effective imaging may include magnetic resonance imaging (MRI), computed tomography $(\mathrm{CT})$, or endoscopic ultrasound. It is recommended that annual biochemical evaluation includes measurement of serum gastrin, glucagon, vasoactive intestinal polypeptide, pancreatic polypeptide, chromogranin A, and insulin levels in addition to a fasting blood glucose. There are few studies evaluating the utility of these peptides in the setting of MEN1 patients. Pancreatic polypeptide (PP), a 36amino-acid peptide produced and secreted by PP cells of the pancreas, has been shown to have a low sensitivity and specificity for pNETs in patients with MEN1, with one study reporting $36 \%$ sensitivity and $74 \%$ specificity [5-7]. However, a separate study found that, in MEN1 patients with elevated 


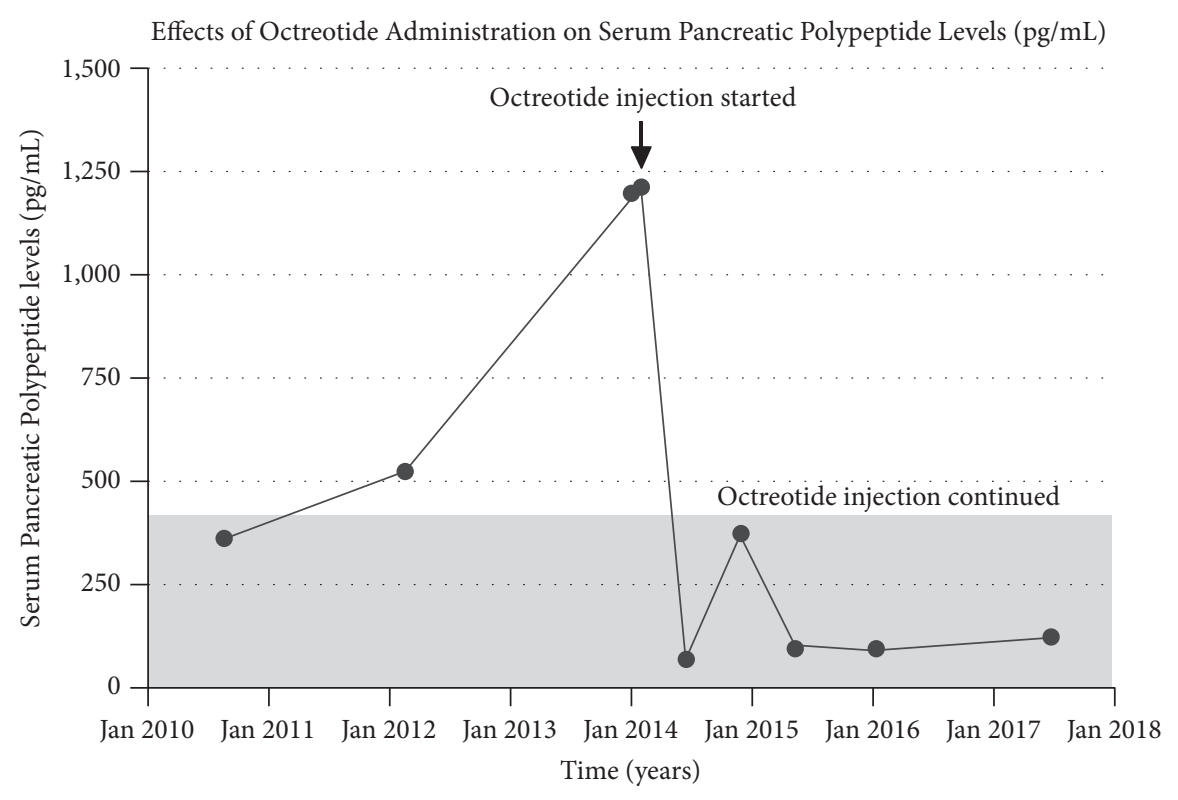

FIGURE 1: Showing serum pancreatic polypeptide (PP) levels (pg/mL) over time. Octreotide treatment was initiated in February 2014. Since initiation of octreotide treatment, the PP levels remain stable. Reference range PP $(0.0-418.0 \mathrm{pg} / \mathrm{mL})$.

PP, a level 3.0 times the normal age-specific value was $95 \%$ sensitive and $88 \%$ specific for an imageable islet cell tumor [7].

The management of pNETs is variable due to the heterogeneity of the disease. The role of surgery for nonfunctional pancreatic tumors (NFPETs) in MEN1 is controversial with the endocrine society clinical practice guideline suggesting surgery for tumors greater than $1 \mathrm{~cm}$ and/or demonstrating significant growth over 6-12 months [1]. For nonresectable tumors, treatment includes somatostatin analogs (SSA), targeted radionucleotide therapy, locoregional treatments, and chemotherapy. Treatment of small pancreatic tumors in asymptomatic individuals has yet to be fully delineated in the guidelines due to lack of long-term data comparing treatment options. Observational data suggest a low potential for metastasis and no increased mortality in NFPETs under $2 \mathrm{~cm}$ [8]. Additionally, pNETs under $2 \mathrm{~cm}$ appear to grow slowly and those under $1 \mathrm{~cm}$ may grow even slower whereas surgical resection at any tumor size can carry substantial risks $[9,10]$.

Here we describe a middle-aged patient with a history of MEN1 who presented with a markedly elevated serum PP level, leading to the discovery of multiple subcentimeter pancreatic lesions found on screening CT, and with prolonged stability on SSA medical therapy.

\section{Case Presentation}

A 43-year-old male reported to endocrine clinic for evaluation of pancreatic lesions. His past history was relevant for total parathyroidectomy for parathyroid hyperplasia, with forearm autograph implantation at the age of 16 years. The patient has a strong family history of pancreatic and parathyroid disorders in his paternal grandfather, two paternal aunts, a paternal uncle, his father, and sister. Additionally, menin gene mutation was confirmed in his father and paternal uncle. The patient underwent a genetic testing at the age of 40 years and this confirmed menin gene mutation. Patient had no symptoms to suggest hypoglycemia, peptic ulcer disease, diarrhea, or other symptoms of endocrine disorders. Review of systems was unremarkable. On physical examination, the vital signs were normal and examination of the heart, lungs, and abdomen was also normal. He has no clinical features of hypogonadism or Cushing syndrome. The patient is married without children and does not smoke or drink alcohol. Additional blood test showed normal serum calcium, testosterone levels, FSH, LH, prolactin, and IGF-I. Additionally evaluation for Cushing syndrome was also negative. Serum gastrin, chromogranin A, vasoactive intestinal polypeptide levels, and 24-hour urine 5-HIAA levels were also normal, and a 48hour fast did not confirm hypoglycemia. However, fasting serum pancreatic polypeptide level was elevated $(520 \mathrm{pg} / \mathrm{mL}$, reference 0 -418). A CT scan of the abdomen showed multiple subcentimeter lesions. The patient refused any treatment initially; 11 months later the serum pancreatic polypeptide levels rose to $1198 \mathrm{pg} / \mathrm{mL}$ (range 912-1588), and 5 weeks later the value was $1215 \mathrm{pg} / \mathrm{mL}$ (Figure 1 ). At this time a repeat CT scan showed 3 hyperenhancing lesions in the head and tail of the pancreas measuring 10, 9, and $4 \mathrm{~mm}$ (Figure 2). These lesions correlated with 111-Indium Octreotide scan (Figure 3). Since the patient refused any surgical treatment, he was offered octreotide treatment. Initially the patient was treated with the short-acting octreotide $100 \mu \mathrm{g} 3$ times daily, and this was later transitioned to a long-acting octreotide $20 \mathrm{mg}$ monthly. Following the octreotide treatment, the serum pancreatic polypeptide levels normalized to $62 \mathrm{pg} / \mathrm{mL}$ in approximately 6 weeks (Figure 1). A follow-up CT scan at 9 and 33 months later confirmed stability of the pancreatic lesions without evidence of metastasis. Additionally, the 


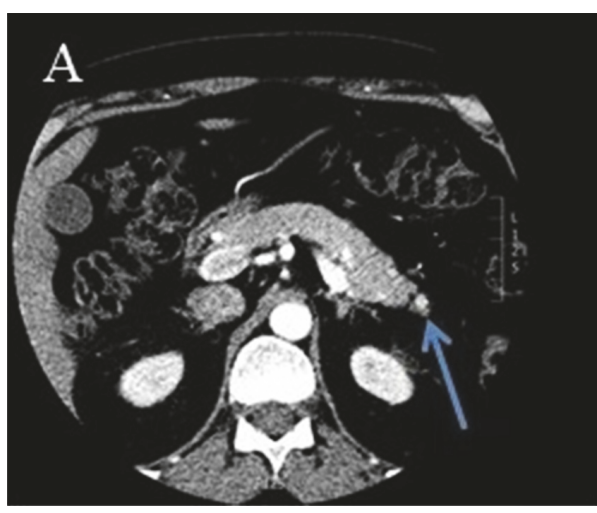

(a)

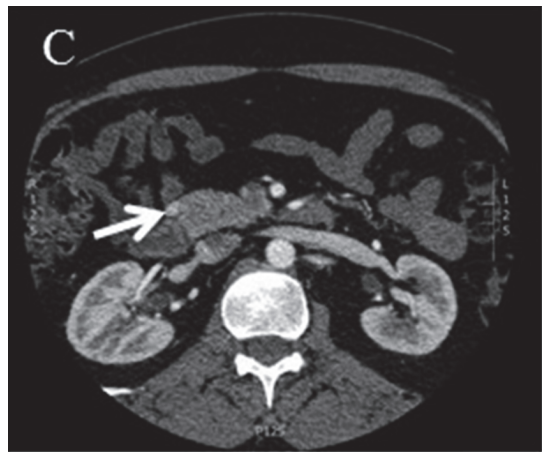

(c)

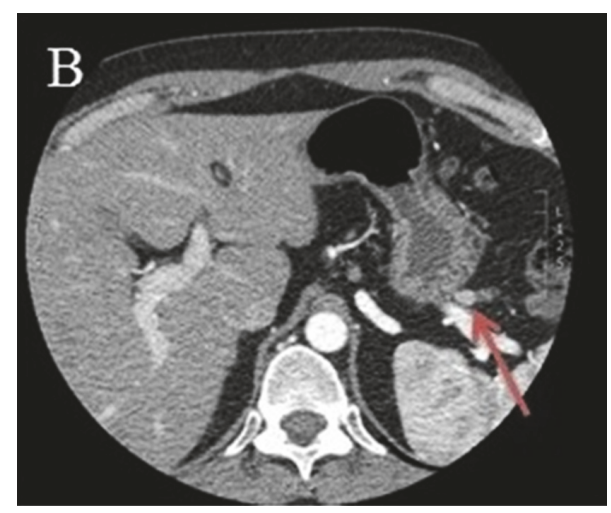

(b)

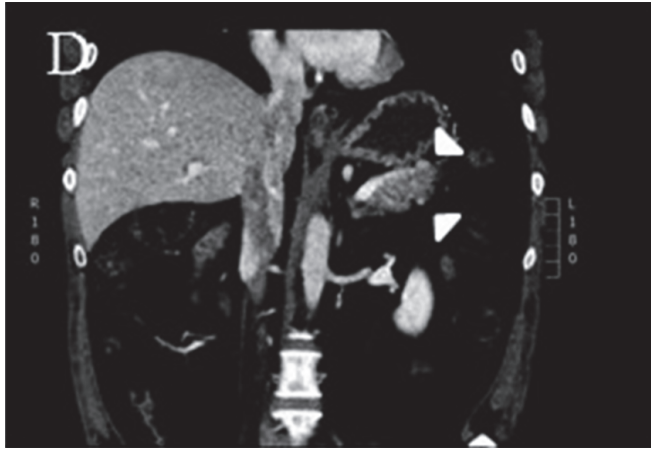

(d)

FIGURE 2: CT of the pancreas showing three hyperenhancing lesions. (a) $4 \mathrm{~mm}$ lesion in the inferior aspect of the pancreatic tail (blue arrow). (b) $9 \mathrm{~mm}$ lesion in the superior aspect of the pancreatic tail (red arrow). (c) $10 \mathrm{~mm}$ lesion in the pancreatic head (white arrow). (d) Coronal view revealing both pancreatic tail lesions (arrow heads).

serum pancreatic polypeptide levels remained within normal limits $(91.6 \mathrm{pg} / \mathrm{mL}$ ) (Figure 1). The patient continued to feel well and has tolerated octreotide without any adverse effects over the entire period of treatment. A most recent gallium68 DOTATATE PET/CT scan (Figure 4) performed 3 years later, while the patient was on octreotide therapy, further confirmed the stability of the pancreatic lesions. Additionally, serum pancreatic polypeptide remained normal along with normal levels of somatostatin, glucagon, gastrin, vasoactive intestinal polypeptide, and chromogranin A during the entire course of octreotide treatment.

\section{Discussion}

Use of somatostatin analogs in the treatment of pNETs is based on investigations showing that the natural 14amino-acid peptide binds to G-protein-coupled somatostatin receptors (SSTRs) that are expressed on most NETs with SSTR-2 expressed in approximately $80 \%$ of pNETs [11, 12]. Octreotide and lanreotide bind with a high affinity to SSTR-2 $[13,14]$. Binding to these receptors can lead to antisecretory and antiproliferative effects. It has been shown that significant improvement in patients with gastrinoma, VIPoma, and glucagonoma can occur following somatostatin analog treatment [15]. In the phase III CLARINET trial of 204 patients, lanreotide $120 \mathrm{mg}$, in patients with advanced, well or moderately differentiated nonfunctional GI and pancreatic NETs with SSTR avid disease, leading to significantly prolonged median progression-free survival (>24 mo.) vs. placebo (18 mo.) [16]. Given the relative good safety profile of somatostatin analog therapy, the results of the CLARINET trial significantly question the utility of observation in patients with locally, advanced, unresectable, or metastatic NETs and support the inclusion of SSA treatment in early stage NET management. National Comprehensive Cancer Network (NCCN) guidelines now state that treatment can be considered for these patients with sporadic NFPETs [17]. Whether this would be the case in an NFPET in MEN1 is unknown. Results from the Dutch MEN1 and the French Endocrine Tumor study groups have recently shown that surgical treatment of NFPET nodules $<2 \mathrm{~cm}$ versus observation resulted in no significant increase in survival over time but did cause a significant number of postoperative complications $[8,18]$. If surgery is no longer a reasonable treatment option for this group of patients and observation is not a reasonable option for a patient, SSA therapy may be an appropriate alternative. The currently available data suggest that there may be a benefit in these small, $<2 \mathrm{~cm}$, nonfunctional nodules. In a single study in twenty MEN1 patients on SSA therapy over 12-75 months of follow-up with tumors $<2 \mathrm{~cm}$, stable disease was found in $80 \%$ of patients, tumor shrinkage in $10 \%$, and hormonal suppression 


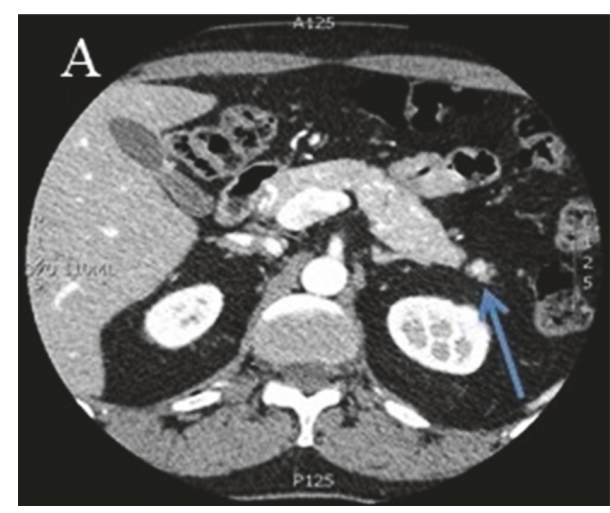

(a)

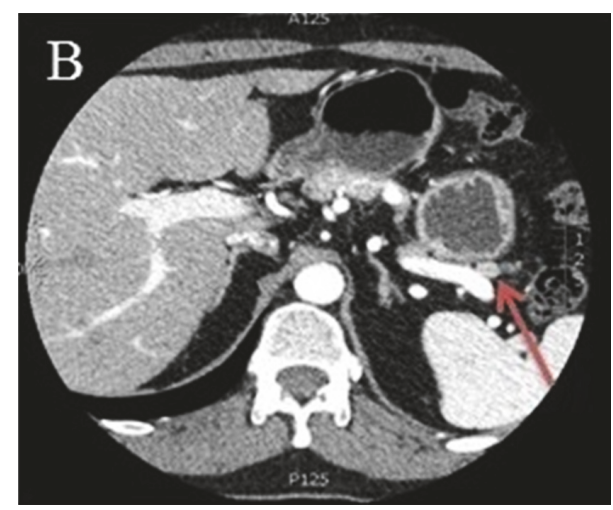

(b)

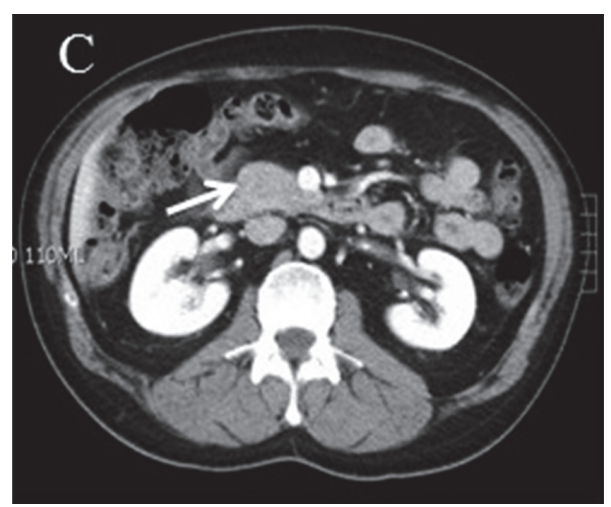

(c)

FiguRE 3: CT of the pancreas showing three hyperenhancing lesions which have remained stable 38 months after initiating octreotide. (a) Stable $4 \mathrm{~mm}$ tumor in the inferior aspect of the pancreatic tail (blue arrow). (b) Stable lesion in the superior aspect of the pancreatic tail (red arrow). (c) Stable lesion in the pancreatic head.

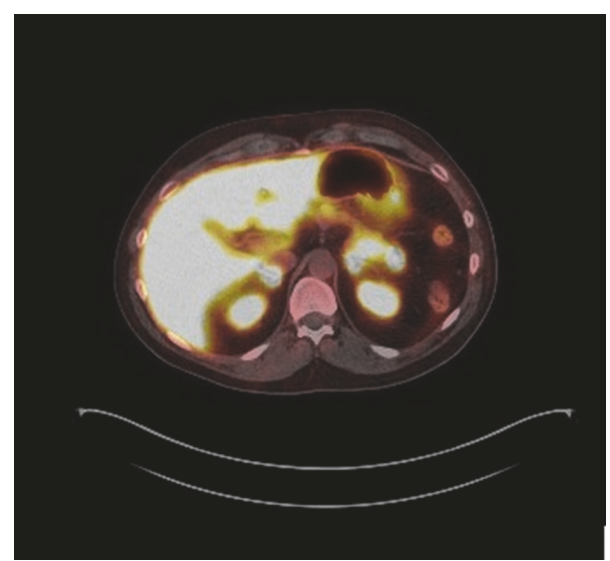

Figure 4: Gallium-68 DOTATATE PET/CT scan showing stability of the pancreatic tumors over time.

in all 6 patients with elevated CgA or gastrin levels [19]. Cioppi et al. (LARO-MEN1 study) investigated whether early treatment with long-acting somatostatin analog could act as a preventive approach in relatively small MEN1-related gastroenteropancreatic neuroendocrine tumors. In 8 patients with small $(<2 \mathrm{~cm})$ neuroendocrine tumors and abnormal laboratory values of at least one of the gastroenteropancreatic hormones, administration of octreotide acetate slow-release formulation (LAR) (10 mg intramuscularly every 28 days) was effective in decreasing gastroenteropancreatic hormone levels and stabilizing tumor size. Furthermore the treatment was safe in the majority of patients up to six years of treatment [20]. Additional data in this select population is likely many years away but a phase 3 trial in Europe comparing SSA therapy vs. no treatment in MEN1 patients with NFPET will be opening soon for enrollment [21].

Additionally, there is insufficient evidence to clarify whether a decline in PP level correlates with a reduction in tumor burden. We would argue that the significant decline in PP level, a nonspecific tumor marker, in addition to the lack of tumor growth and stable follow-up PP level is indicative of a treatment effect as has been seen with other hormonally active NETs treated with SSA therapy. We speculate that if the level of PP continues to rise off therapy, the patient would experience further nodular growth, which could have adverse health consequences. Typical management of subcentimeter NFPETs has typically involved serial imaging and biochemical testing. SSA therapy in addition to serial testing represents a reasonable treatment option that is well tolerated in this patient population although its high-cost may hinder its utilization. 
Furthermore, basal and meal-stimulated pancreatic polypeptide has been shown to be useful for early detection of pancreatic involvement in $75 \%$ of patients with MEN-1. Our patient's basal fasting pancreatic polypeptide levels were not only elevated but actually doubled at one-year followup. The stability of the pancreatic tumors along with the normalization of serum PP level confirms the effectiveness of SSA therapy in this patient. It may be stated that the pancreatic lesions would have remained stable without octreotide treatment; however, octreotide was effective in our patient for several reasons: (1) the serum PP level doubled over the 11month observation period when he did not receive octreotide treatment; (2) there was an increase in the size of pancreatic tumors during this observation period; and (3) a gallium68 DOTATATE scan showed stability of tumor size over the course of treatment. Therefore, these findings support the effectiveness of octreotide treatment. As the benefits of pancreatic surgery for small lesions $(<2 \mathrm{~cm})$ are not clear, the morbidity, mortality, and long-term complications of surgery must be considered. Our patient was thus offered medical therapy with long-acting octreotide, which was recently shown to provide $90 \%$ objective tumor response and stability in MEN-1 patients with early nonfunctional pancreatic NET [19]. Discontinuation of octreotide and reassessment of serum pancreatic polypeptide level and tumor growth would have confirmed the effectiveness of octreotide therapy; however, we felt it may be unethical to discontinue octreotide therapy.

\section{Conclusion}

The malignant potential of pNETs is the primary lifethreatening manifestation of MEN1 but treatment for NFPET $<1 \mathrm{~cm}$ has not been clearly defined by the guidelines. We describe the case of a middle-aged patient with a history of MEN1 who presented with elevated serum PP levels and multiple subcentimeter pancreatic lesions found on imaging who was treated with SSA therapy for 24 months leading to a marked reduction in PP level during treatment with stability in lesion size after 40 months of follow-up. SSA therapy represents a reasonable and potential alternative to watchful waiting in this patient population but further research is necessary to better define long-term outcomes.

\section{Abbreviations}

CgA: Chromogranin A

CT: Computed tomography

MEN1: Multiple endocrine neoplasia type 1

NFPET: Nonfunctional pancreatic tumor

pNET: Pancreatic neuroendocrine tumor

PP: $\quad$ Pancreatic polypeptide

VIP: Vasoactive intestinal peptide

PET: Positron emission tomography.

\section{Disclosure}

We certify that the document represents valid work; that if we used information derived from another source, we obtained all necessary approvals to use it and made appropriate acknowledgments in the document; and that each author takes public responsibility for it. The authors are employees of the United States Government. This work was prepared as part of their official duties. Title 17 U.S.C. $\$ 105$ provides that 'Copyright protection under this title is not available for any work of the United States Government.' Title 17 U.S.C. $\$ 101$ defines a U.S. Government work as a work prepared by a military service member or employee of the U.S. Government as part of that person's official duties. The views expressed in this article are those of the authors and do not necessarily reflect the official policy or position of the Department of the Navy, the Department of Defense, nor the U.S. Government.

\section{Conflicts of Interest}

The authors have no conflicts of interest to disclose.

\section{Authors' Contributions}

All individuals who qualify as authors have been listed; each has participated in the conception and design of this work, the analysis of data, the writing of the document, and the approval of the submission of this version.

\section{Acknowledgments}

We would like to thank Mr. Jay McDaniel for preparing the figure in this paper.

\section{References}

[1] R. V. Thakker, P. J. Newey, G. V. Walls et al., "Clinical practice guidelines for multiple endocrine neoplasia type 1 (MEN1)," The Journal of Clinical Endocrinology \& Metabolism, vol. 97, no. 9, pp. 2990-3011, 2012.

[2] T. Ito, H. Igarashi, H. Uehara, M. J. Berna, and R. T. Jensen, "Causes of death and prognostic factors in multiple endocrine neoplasia type 1: A prospective study:Comparison of 106 MEN1/zollinger-ellison syndrome patients with 1613 literature menl patients with or without pancreatic endocrine tumors," Medicine (United States), vol. 92, no. 3, pp. 135-181, 2013.

[3] F. Triponez, D. Dosseh, P. Goudet et al., "Epidemiology data on 108 MEN 1 patients from the GTE with isolated nonfunctioning tumors of the pancreas," Annals of Surgery, vol. 243, no. 2, pp. 265-272, 2006.

[4] M. A. Kouvaraki, S. E. Shapiro, G. J. Cote et al., "Management of pancreatic endocrine tumors in multiple endocrine neoplasia type 1," World Journal of Surgery, vol. 30, no. 5, pp. 643-653, 2006.

[5] W. Qiu, I. Christakis, A. Silva et al., "Utility of chromogranin A, pancreatic polypeptide, glucagon and gastrin in the diagnosis and follow-up of pancreatic neuroendocrine tumours in multiple endocrine neoplasia type 1 patients," Clinical Endocrinology, vol. 85 , no. 3, pp. 400-407, 2016.

[6] J. M. de Laat, C. R. Pieterman, M. Weijmans et al., "Low accuracy of tumor markers for diagnosing pancreatic neuroendocrine tumors in multiple endocrine neoplasia type 1 patients," The Journal of Clinical Endocrinology \& Metabolism, vol. 98, no. 10, pp. 4143-4151, 2013. 
[7] M. G. Mutch, M. M. Frisella, M. K. DeBenedetti et al., "Pancreatic polypeptide is a useful plasma marker for radiographically evident pancreatic islet cell tumors in patients with multiple endocrine neoplasia type 1," Surgery, vol. 122, no. 6, pp. 10121020, 1997.

[8] F. Triponez, S. M. Sadowski, F. Pattou et al., "Long-term Followup of MEN1 patients who do not have initial surgery for small $\leq 2 \mathrm{~cm}$ nonfunctioning pancreatic neuroendocrine tumors, an AFCE and GTE study: Association Francophone de Chirurgie Endocrinienne \& Groupe d'Etude des Tumeurs Endocrines," Annals of Surgery, vol. 268, pp. 158-164, 2018.

[9] W. F. W. Kappelle, G. D. Valk, M. Leenders et al., "Growth rate of small pancreatic neuroendocrine tumors in multiple endocrine neoplasia type 1: Results from an endoscopic ultrasound based cohort study," Endoscopy, vol. 49, no. 1, pp. 27-34, 2017.

[10] Y. N. You, G. B. Thompson, W. F. Young Jr. et al., "Pancreatoduodenal surgery in patients with multiple endocrine neoplasia type 1: Operative outcomes, long-term function, and quality of life," Surgery, vol. 142, no. 6, pp. 829-836, 2007.

[11] M. Papotti, M. Bongiovanni, M. Volante et al., "Expression of somatostatin receptor types 1-5 in 81 cases of gastrointestinal and pancreatic endocrine tumors. A correlative immunohistochemical and reverse transcriptase polymerase chain reaction analysis," Virchows Archiv, vol. 440, no. 5, pp. 461-475, 2002.

[12] J. C. Reubi, B. Waser, J.-C. Schaer, and J. A. Laissue, "Somatostatin receptor sst1-sst5 expression in normal and neoplastic human tissues using receptor autoradiography with subtypeselective ligands," European Journal of Nuclear Medicine and Molecular Imaging, vol. 28, no. 7, pp. 836-846, 2001.

[13] W. Bauer, U. Briner, W. Doepfner et al., "SMS 201-995: a very potent and selective octapeptide analog of somatostatin with prolonged action," Life Sciences, vol. 31, no. 11, pp. 1133-1140, 1982.

[14] H. Shojamanesh, F. Gibril, A. Louie et al., "Prospective study of the antitumor efficacy of long-term octreotide treatment in patients with progressive metastatic gastrinoma," Cancer, vol. 94, no. 2, pp. 331-343, 2002.

[15] R. Eldor, B. Glaser, M. Fraenkel, V. Doviner, A. Salmon, and D. J. Gross, "Glucagonoma and the glucagonoma syndrome - Cumulative experience with an elusive endocrine tumour," Clinical Endocrinology, vol. 74, no. 5, pp. 593-598, 2011.

[16] M. E. Caplin, M. Pavel, J. B. Ćwikła et al., "Lanreotide in metastatic enteropancreatic neuroendocrine tumors," The New England Journal of Medicine, vol. 371, no. 3, pp. 224-233, 2014.

[17] M. H. Kulke, M. H. Shah, A. B. Benson 3rd et al., "Neuroendocrine tumors," Journal of the National Comprehensive Cancer Network, vol. 13, pp. 78-108, 2015.

[18] S. Nell, H. M. Verkooijen, C. R. C. Pieterman et al., "Management of MEN1 related nonfunctioning pancreatic NETs: A shifting paradigm: results from the DutchMEN1 study group," Annals of Surgery, vol. 267, no. 6, pp. 1155-1160, 2018.

[19] V. Ramundo, M. Del Prete, V. Marotta et al., "Impact of long-acting octreotide in patients with early-stage MEN1related duodeno-pancreatic neuroendocrine tumors," Clinical Endocrinology, vol. 80, no. 6, pp. 850-855, 2014.

[20] F. Cioppi, L. Cianferotti, L. Masi et al., "The LARO-MEN1 study: a longitudinal clinical experience with octreotide Long-Acting Release in patients with Multiple Endocrine Neoplasia type 1 Syndrome," Clinical Cases in Mineral and Bone Metabolism, vol. 14, no. 2, pp. 123-130, 2017.

[21] A. Selberherr, Non-functioning pancreatic neuroendocrine tumors in MEN1: somatostatin analogs versus no treatment (SANO),
2018, https://clinicaltrials.gov/ct2/show/NCT02705651?cond= Non-functioning+Pancreatic+Neuroendocrine+Tumors+in+ MEN1\&amp;rank=1. 


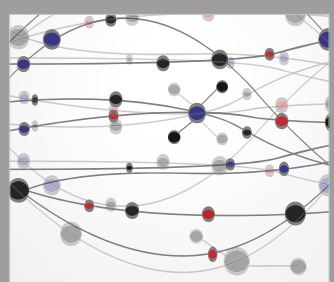

The Scientific World Journal
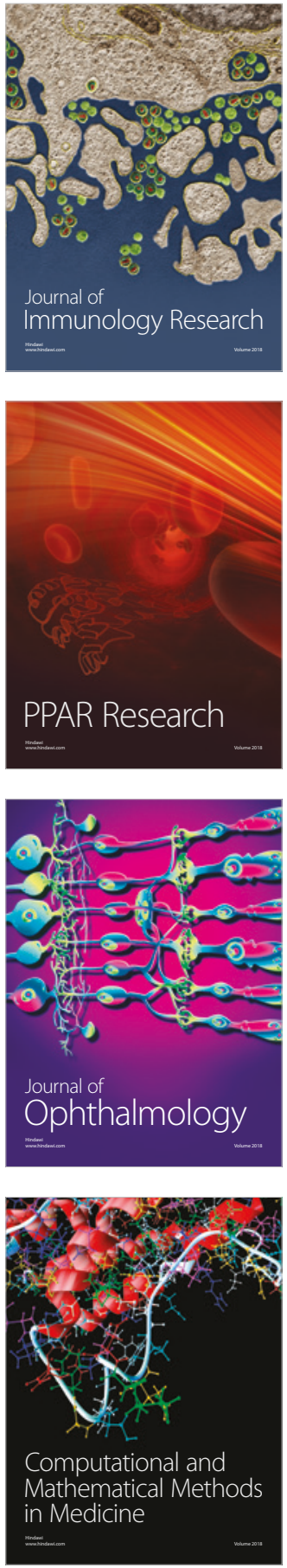

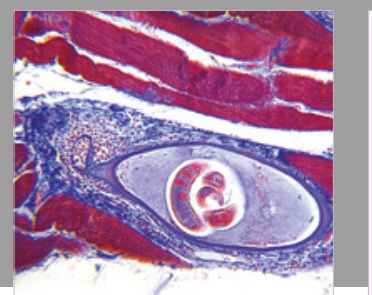

Gastroenterology Research and Practice

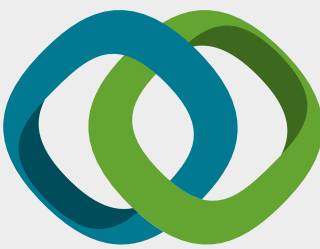

\section{Hindawi}

Submit your manuscripts at

www.hindawi.com
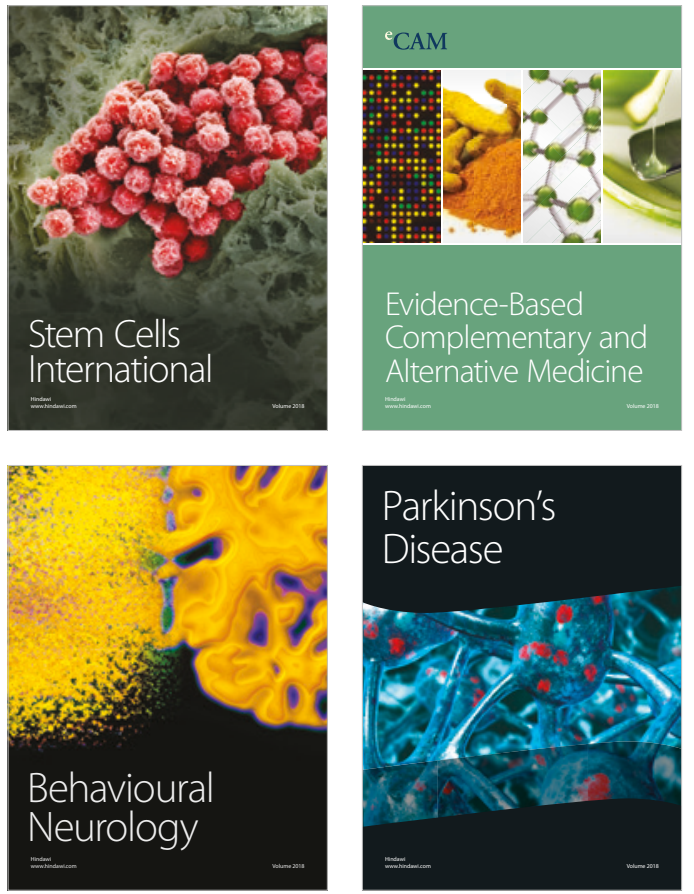

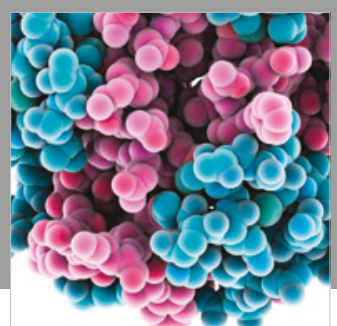

ournal of

Diabetes Research

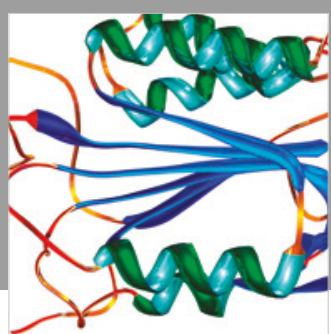

Disease Markers
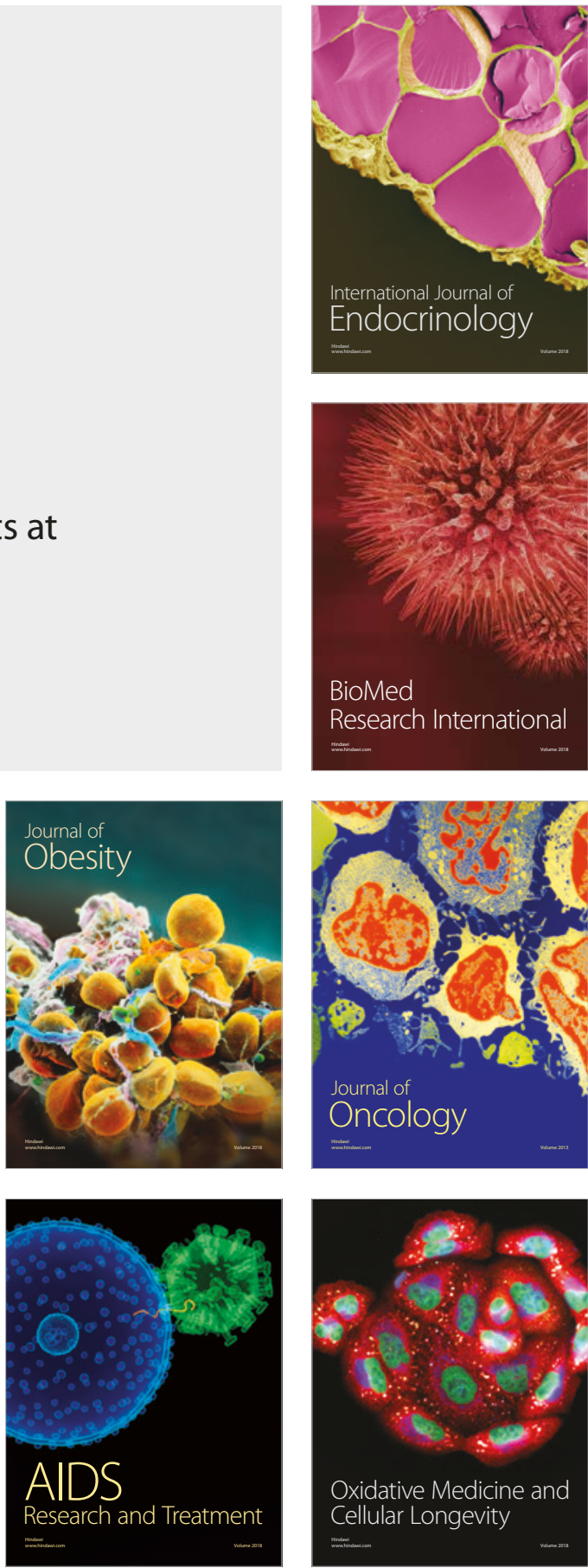